\title{
Navigating the choppy waters of change
}

\section{Commentary}

Communication, collaboration and team are words that healthcare endorses, and evidence supports, as best practice in providing quality, safe and reliable care. ${ }^{1}$ Collaborative practice, multi-disciplinary or interdisciplinary teams ${ }^{2}$ have demonstrated better quality care for patients for decades, but in order to be successful, some foundational elements are required to what in essence is effective team functioning. Yet, putting into practice these words is not always easy or well applied despite best intentions. In the background of the delivery of patient care lies the operational culture between leaders and staff who strive towards effective team functioning.

In today's healthcare world, it is akin to a boat staying afloat amidst a tsunami of change whether they are from external forces such as innovative technologies, treatments or internal process and procedure change, it is becoming increasingly difficult to put an operational best foot forward. In addition to this already tumultuous environment, the introduction of roles such as licensed practical nurses (LPN), medical office assistant (MOA) and in-scope leadership roles such as a nurse clinician (NC) into an area of traditionally homogenous frontline roles (registered nurses) elicits emotional forces of uncertainty and anxiety by staff and leaders creating more churn.

One example of such an area is ambulatory oncology centers that have provided care within multi-disciplinary teams. Those multidisciplinary teams are comprised of different physician disciplines and allied health, yet primary registered nurses with clerical support staff have predominantly led the clinical case management. Due to expanding scopes of practice as well as ensuring that the 'right person is looking after the right patient at the right time', collaborative practice is making its way to the outpatient setting challenging the notion that care can only be within one discipline's purvey.

In 2015, a MOA and 4 NC's were introduced into an ambulatory oncology centre to help support both the patient and staff experience, as well as to enhance the in-scope leadership opportunities to move change forward. A study interviewing 15-20 staff members affected by the introduction and integration of these new roles is underway. Preliminary results point to the culture chasm between pre and post-merger of 12 health regions ${ }^{3}$ into one in 2008. Interactive communication and collaboration before these roles were introduced (including role clarity and opportunity for sense-making and support), were requirements for successful role introduction and integration. Interestingly though, two years later questions still remain as to the why, what and how of these new roles yet there is apathy towards asking questions or clarifying issues. Consequently incorrect
Volume 5 Issue $6-2017$

\author{
Carol Baumgarten,' Ron Fisher ${ }^{2}$ \\ 'Director Cancer Care Teams, Alberta Health Services, Canada \\ ${ }^{2}$ Griffith University, Australia
}

Correspondence: Ron Fisher, Griffith University, Australia, Email r.fisher@griffith.edu.au

Received: May 29, 2017 | Published: May 30, 2017

perceptions remain about these roles. The overarching post-merger culture, and the seemingly incongruent way of doing business by leaders and the organization, suggests that basing change on this shaky foundation of ineffective understanding and expectation needs to be reset with current expectations and standards and a go-forward created.

\section{Concluding thoughts}

Communication, collaboration and team building do not happen by accident. They require a foundation of well-managed expectations, respect, compassion, and openness to start laying the platform to trust. In this way a culture of acceptance and constructive collaboration can be created in order to develop an effective operational team.

\section{Acknowledgements}

None.

\section{Conflict of interest}

Author declares that there is no conflict of interest.

\section{References}

1. Borras JM, Albreht T, Audisio R, et al. Policy statement on multidisciplinary cancer care. Eur J Cancer. 2014;50(3):475-480.

2. Al Sayah F, Szafran O, Robertson S, et al. Nursing perspectives on factors influencing interdisciplinary teamwork in the Canadian primary care setting. J Clin Nurs. 2014;23(19-20):2968-2979.

3. AHS. 2017. 